\title{
Comparison of Intra-Articular versus Intravenous Application of Tranexamic Acid in Total Knee Arthroplasty: A Prospective Randomized Study
}

\author{
Kornah BA, Mansour GM and Mohamed MTI* \\ Orthopedic Surgery Department, Faculty of Medicine, Al- \\ Azhar University, Egypt \\ *Corresponding author: Mohamed Tawfiq Ibrahim \\ Mohamed, Orthopedic Surgery Department, Faculty of \\ Medicine, Al-Azhar University, Egypt
}

Received: May 07, 2021; Accepted: J une 07, 2021;

Published: June 14, 2021

\begin{abstract}
Background: Total Knee Arthroplasty (TKA) is widely used in clinical applications. Considerable postoperative blood loss, however, is an unavoidable occurrence due to the extensive soft-tissue release, and the large area of cancellous bone osteotomy associated with the procedure. Besides, knee flexion and extension activities those begin the day after the operation adds to the problem.
\end{abstract}

Aim of the Work: To compare between the intra-articular and the intravenous application of Tranexamic Acid (TXA) in total knee arthroplasty.

Patients and Methods: It is a prospective Double-blinded Randomized controlled trial. This prospective study was conducted at El-Hussein University Hospital \& El Agouza Hospital on 30 patients who undergo total knee arthroplasty and fulfill all inclusion and exclusion criteria.

Results: The results were the average total blood loss in the drain after $48 \mathrm{hrs}$. In the $1^{\text {st }}$ group was $365 \pm 74.722$ while average blood loss in the $2^{\text {nd }}$ group was $575 \pm 88.976$. This indicates a significant decrease in blood loss in the $1^{\text {st }}$ group (intraarticular TXA group) than the $2^{\text {nd }}$ group (intravenous TXA group) the $P$ value $<0.001$ which is highly significant. It was found that The decrease in $\mathrm{Hb}$ level is less in the $1^{\text {st }}$ group than in the $2^{\text {nd }}$ group which indicates less postoperative anemia in the blood count, the P-value $<0.05$ considered significant.

Conclusion: Concerning intra-articular administration of TXA seemed to be more effective than intravenous injection in terms of blood loss and transfusion frequency.

Keywords: Total knee arthroplasty; Tranexamic acid; Blood loss

\section{Introduction}

Total Knee Arthroplasty (TKA) is an effective treatment for the advanced disease of the knee joint, which can relieve pain and improve joint function. With improvements in surgical techniques and prosthetic design. TKA is a widely used clinical application. Considerable postoperative blood loss, however, is an unavoidable occurrence due to the extensive soft-tissue release, and a large area of cancellous bone osteotomy associated with the procedure. Also, knee flexion and extension activities that begin the day after the operation adds to the problem. Postoperative dominant blood loss after unilateral TKA can reach 1,200-1,900 mL [1].

A growing number of elderly patients undergo TKA. This patient group can suffer from many underlying diseases, such as an impaired compensatory ability of the cardiovascular and hematopoietic systems. As such, hemorrhage can be a serious consequence. Anemia is a key impeding factor in patients' postoperative recovery. Homologous blood transfusion is often used to solve postoperative anemia, but this carries a substantial risk of immunologic reaction and disease transmission for the patients and also increases medical expenses [2]. Therefore, a reduction in operative blood loss, especially post TKA, is beneficial to recovery and decreases the risk of transfusion. The use of pneumatic tourniquets leads to an increase in the activity of the fibrinolytic system (a naturally existing enzymatic system that regulates clot and thrombus formation in the body). Paradoxically, this may accentuate surgical blood loss [3-5].

Various measures have been tried to decrease the bleeding and subsequently, the transfusion requirements, such as the use of antifibrinolytic agents $[6,7]$.

Tranexamic Acid (TXA) which is a synthetic derivative of amino acid lysine and a competitive inhibitor of plasminogen activation and thus interferes with fibrinolysis by competing with the lysine molecule at fibrinogen binding sites has demonstrated in having the ability to reduce blood loss, but there is still controversy regarding the dosage and the route of administration [8].

\section{Aim of the Work}

The objective of this randomized comparative study was to compare the intra-articular and intravenous application of Tranexamic Acid (TXA) in total knee arthroplasty, regarding the volume of blood loss and change in $\mathrm{Hb}$ level preoperatively and 48 
hours postoperatively.

\section{Patients and Methods}

It is a prospective Double-blinded Randomized controlled trial This perspective was conducted at El Hussein University Hospital \& El Agouza Hospital.

This prospective randomized control study was conducted on 30 patients who undergo total knee arthroplasty and fulfill all inclusion and exclusion criteria.

\section{Inclusion criteria}

- Patients undergoing unilateral TKR for primary osteoarthritis \& rheumatoid arthritis.

- $\quad$ Age above 55;

- The same approach was used for all cases (standard midline incision with medial parapatellar).

\section{Exclusion criteria}

Patients presenting one or more of the following criteria were excluded from the study:

- History or identified risk of deep venous thrombosis or pulmonary embolism.

- History of coagulation or cardiovascular disorders; vascular diseases.

- Current use of anticoagulant drugs.

- Orthopedic surgery in the lower limbs.

- Consent from a refusal.

- Contra-indication for TXA uses (active intravascular clotting process, acquired defective color vision, subarachnoid hemorrhage, and hypersensitivity to TXA).

\section{Patients}

Preoperative patient evaluation: All the patients are above 55 years old suffering from severe osteoarthritis in the knee joint which failed to be treated by the medication or even by intraarticular injections.

History taking: A detailed history was obtained about the patients' symptoms and their duration. All the patients complained of severe pain in the knee especially on flexion and difficulty walking more than 20 meters without stop from pain.

Full present history is taken from patients smoking, hypertension, diabetes, cardiac, previous operations, and previous DVT and regular drugs taken by the patients.

For Cardiac patients who are taking aspirin $75 \mathrm{mg}$, aspirin was stopped 5 days before operation day and shifted on low molecular weight heparin (LMWH; Clexane 40mg).

Clinical examination: All patients had a full examination for the knee joint, tenderness and inability to flex the knee was presented in all patients, furthermore, a flexion deformity or valgus deformity is found in some patients, besides that some of them had visible baker cyst in the posterior aspect of the knee.

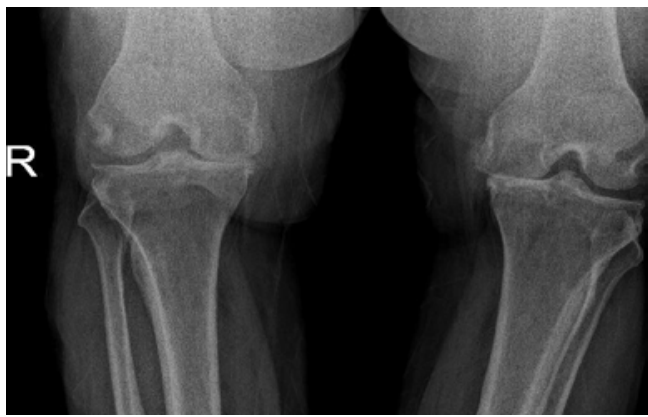

Figure 1: X-ray P.A view both knees standing position.

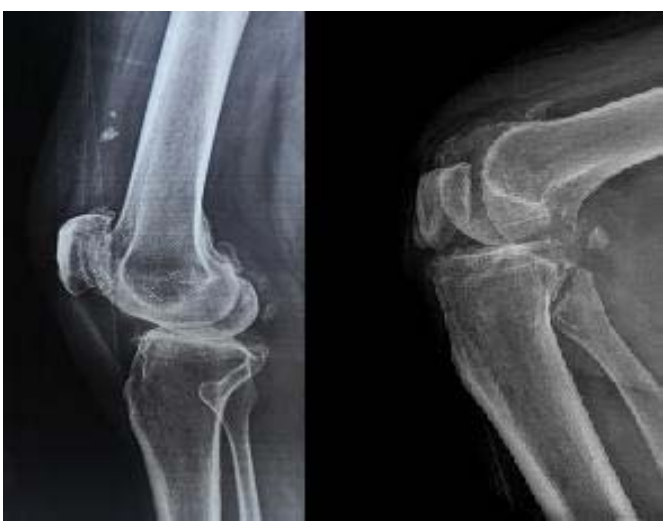

Figure 2: X-ray of both knee lateral views.

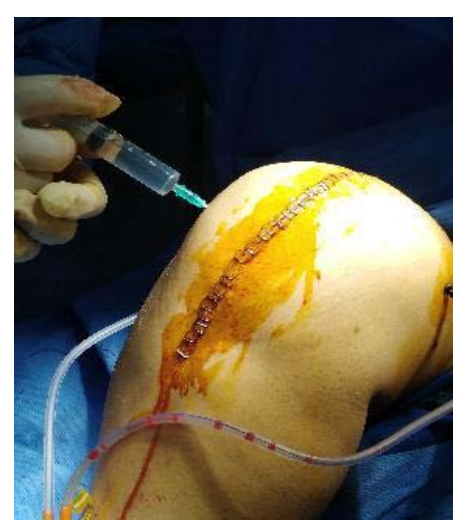

Figure 3: Intra-articular tranexamic acid injected into the knee joint cavity after the closure of the joint capsule.

Lab investigation: Preoperative full laboratory investigation is done for all patients; complete blood count, prothrombin time, partial thromboplastin time, INR, fasting and postprandial blood sugar, ESR, CRP, ALT, AST, and kidney functions were done.

Radiographic evaluation: All the patients had done X-ray standing position on both knee joints Anteroposterior and normal $\mathrm{x}$-ray lateral view (Figure 1 and 2).

\section{Methods}

Thirty patients undergone Total knee replacement divided into three groups: 
The first group: 10 cases had intra-articular tranexamic acid (1.5g injected into the knee joint cavity after the closure of the joint capsule) (Figure 3).

The second group: 10 cases received intravenous tranexamic acid $(15 \mathrm{mg} / \mathrm{kg}$ in $100 \mathrm{~mL}$ of saline single dose at the start of operation).

The third group: 10 cases injected with saline only.

Anesthesia: All patients were operated on under epidural anesthesia, with Tourniquet control of $350 \mathrm{Hg}$ pressure.

Crystalloids in the form of Normal Saline (NS) were used as maintenance fluid; also plasma of the same blood group of the patient was used in some patients as maintenance fluid.

All patients were positioned in the supine position.

Surgical technique: A standard midline incision with a medial Para patellar approach was used for all the surgeries.

Standard techniques of intraoperative hemostasis were used. All knees implanted with a Posterior Stabilized (PS) cemented knee (Zimmer). Wounds are closed in layers over the non- suction drain and the drain is closed for 2 hours.

Postoperative protocol: A similar protocol for the groups was used. Postoperative note for nurse staff to leave the drain closed for 2 hours after the operation and continuous reading for the drains, the amount was recorded in the patient file. A postoperative X-ray on the knee was requested

Complete blood count was done 48 hours after surgery to evaluate $\mathrm{Hb}$ level postoperatively.

Antibiotics used for prophylaxis are broad-spectrum covering Gram +ve and -ve bacteria. They were given as I.V injections during 5-7 days' hospital stay then the patient was shifted on oral antibiotic till the first visit which was 10 days postoperatively.

Standard prophylaxis against venous thromboembolism in the form of subcutaneous injections of low molecular weight heparin (LMWH) is given to all patients once daily from day 1 till 21 days postoperatively.

During the first visit to the outpatient clinic evaluation of joint movement, skin edema, and clinical evaluation for the presence of DVT, removal of the stitches, and X-ray (Ap, lateral views) were done.

Postoperative evaluation: Complete blood count was done 48 hours after surgery to evaluate $\mathrm{Hb}$ level postoperatively.

Total draining fluids in the drain are documented for 48 hours according to the recorded amount in the patient file. All the results were collected in a personal notebook to be used in statistical analysis.

Also, an evaluation for DVT clinically is done during the patient stay in the hospital.

\section{Statistical analysis}

Data were analyzed using Statistical Program for Social Science. IBM SPSS Statistics Subscription. Quantitative data were expressed as mean \pm standard deviation (SD). Qualitative data were expressed as frequency and percentage.

The following tests were done:
- Independent-samples t-test of significance was used when comparing between two means.

- Paired sample t-test of significance was used when comparing between related samples.

- Chi-square (X2) test of significance was used to compare proportions between two qualitative parameters.

- Probability (P-value).

1. P-value $<0.05$ was considered significant.

2. P-value $<0.001$ was considered as highly significant.

3. P-value $>0.05$ was considered insignificant.

\section{Results}

\section{Demographic features}

The patients' age, sex, side of the operation and co-morbidity (hypertension, smoking, diabetes) are shown in Table 1:

- 18 patients are females (65.0\%) and 12 patients are males (35.0\%).

- 6 patients are smokers (20.0\%) and all are males.

- All the patients are above 55 years old (58 years to 68 years) with an average of $(63 \pm 3.212)$

- 21 patients are diabetic $(70.0 \%)$, all controlled on oral hypoglycemic drugs.

- 15 patients are hypertensive (50.0\%), all controlled on oral antihypertensive drugs.

- 9 patients underwent left TKR (30.0\%) and 21 had Right TKR

Table 1: All parameters descriptive of the study group.

\begin{tabular}{|c|c|}
\hline & No. (\%) $(\mathbf{N}=\mathbf{2 0})$ \\
\hline Fex & $18(60.0 \%)$ \\
\hline Male & $12(40.0 \%)$ \\
\hline Age (years) [Range (Mean \pm SD) $]$ & {$[58-68(63 \pm 3.212)]$} \\
\hline Smoker & $6(20.0 \%)$ \\
\hline Diabetics & $21(70.0 \%)$ \\
\hline Hypertension & $15(50.0 \%)$ \\
\hline Side of TKR & $9(30.0 \%)$ \\
\hline Left & $21(70.0 \%)$ \\
\hline Right & \\
\hline
\end{tabular}

${ }^{*} \mathrm{P}$-value is calculated by comparing the three groups.

${ }^{*}$ Data is presented as Mean \pm SD (range).

Table 2: Comparison between Preoperative and Postoperative according to HB in each group.

\begin{tabular}{|c|c|c|c|}
\hline HB. & Group 1 & Group 2 & Group 3 \\
\hline Preoperative & $12.31 \pm 0.73$ & $12.56 \pm 0.28$ & $12.88 \pm 0.53$ \\
\hline Postoperative & $10.54 \pm 0.47$ & $10.23 \pm 0.53$ & $10.20 \pm 0.68$ \\
\hline Mean Hb drop & 1.77 & 2.33 & 2.68 \\
\hline Independent Samples Test & & 3.265 & \\
\hline P-value & & 0.004 & \\
\hline
\end{tabular}


Table 3: Comparison according to total Redivac drainage in CC.

\begin{tabular}{|c|c|c|c|c|}
\hline Total Redivac Drainage in CC & Group 1 & Group 2 & Group 3 \\
\hline Mean \pm SD & $365 \pm 74.722$ & $575 \pm 88.976$ & $1280.00 \pm 75.12$ \\
\hline Range & $250-500$ & $400-700$ & $1150-1450$ \\
\hline
\end{tabular}

$(70.0 \%)$

There were no statistically significant differences regarding demographic data (age, sex), co-morbidity (hypertension, smoking, diabetes), and side of the operation between the two groups ( $\mathrm{p}$-value $\geq 0.05$ ).

\section{Comparison According to outcomes}

Comparison between the $\mathrm{Hb}$ level preoperatively and 48 hours postoperatively: For the $1^{\text {st }}$ group, the average preoperative $\mathrm{Hb}$ was $12.31 \pm 0.73$, while the postoperative $\mathrm{Hb}$ average was $10.54 \pm 0.47$, with a paired difference of 1.77 .

The $2^{\text {nd }}$ group average preoperative $\mathrm{Hb}$ was $12.56 \pm 0.28$ while the average postoperative $\mathrm{Hb}$ was $10.23 \pm 0.53$, with a paired difference of 2.33 ,

In the $3^{\text {rd }}$ group, the average preoperative $\mathrm{Hb}$ was $12.88 \pm 0.53$, while the postoperative $\mathrm{HB}$ average was $10.20 \pm 0.68$, with a paired difference of 2.68 as shown in Table 2 .

This table shows a highly statistically significant difference between preoperative and postoperative according to $\mathrm{Hb}$ in each group.

Comparison in the total amount of blood loss in the drain 48 hours postoperatively between the three groups: The average total blood loss in the drain after $48 \mathrm{hrs}$. In the $1^{\text {st }}$ group was $365 \pm 74.722$ and in the $2^{\text {nd }}$ group was $575 \pm 88.976$ while in the $3^{\text {rd }}$ was $1280.00 \pm 75.12$ as shown in Table 3.

This table shows a highly statistically significant difference between groups according to total Redivac drainage in CC.

\section{Patient evaluation}

Complete blood count was done 48 hours after surgery to evaluate $\mathrm{Hb}$ level postoperatively.

Total draining fluids in the drain are documented for 48 hours according to the recorded amount in the patient file.

Clinical evaluation for DVT before patient discharge.

\section{Complications}

DVT: No cases had reported any signs of DVT.

Infection: No cases had reported fever or any signs of infection.

\section{Discussion}

One of the main problems after TKA is blood loss which is a must and promotes the need for blood transfusion with its complication [9]. Because TKR is usually performed under tourniquet, veins of the lower extremity are in a state of hypoxia. This results in the release of tissue plasminogen activator from the vascular endothelium, which promotes fibrinolysis. Tourniquet deflation results in sudden vein expansion in the lower extremity, which leads to increased bleeding [10].
The most important finding in this study is that TXA reduces blood loss in TKR and the need for transfusion accordingly. Comparing two TXA groups, intra-articular injection of TXA seems to be more effective than intravenous injection in terms of the amount of blood loss and the need for transfusion.

Intra-articular injection at the surgical site provides direct and straightforward means of application before tourniquet releasing [11]. Also, intra-articular TXA injection has the advantage of inducing partial microvascular hemostasis by stopping fibrin clot dissolution in the affected area. Once injected intra-articularly, TXA is rapidly absorbed and maintains a biological half-time of approximately $3 \mathrm{~h}$ within joint fluid [12].

A concern exists on the use of TXA that the anti-fibrinolytic effect of TXA may cause thromboembolic complications in patients given TKA [13]. Due to the concern, patients with a history of a cardiac problem or with a thromboembolic disease were excluded from this study enrolment. Furthermore, no thromboembolic complications such as DVT or pulmonary embolism have been encountered after intra-articular injection of TXA.

In comparison with other literature; several studies proving the efficacy of both intra- articular and intravenous TXA in reducing blood loss after TKR, the ideal route of administering TXA will remain a topic for ongoing debate and controversy in the upcoming years.

Intra-articular application of TXA has been investigated by many authors in recent years [14]. As the drugs are applied predominantly to the joint cavity, the site of bleeding could achieve a higher therapeutic concentration. This could effectively limit blood loss with little or no systemic absorption or subsequent systemic side effects. Additionally, TXA is easy to administer in this way.

Other recent studies also concluded that the two routes of administration are comparable for blood transfusion incidences after TKR [14]. Their findings support the postulation that the therapeutic effect of TXA becomes apparent when proteolysis of plasmin prematurely dissolves the fibrin clot. As TXA works at the active bleeding site of the wound rather than within the blood vessels, its presence within the clot is considered effective irrespective of the route of administration. However, two recent studies favored intraarticular TXA to intravenous administration [15], while another study reported significantly lower blood transfusion incidence with intravenous TXA [16].

Wong et al. [17] compared 150 patients undergoing primary unilateral total knee arthroplasty were randomly distributed to 3 groups (IV, intra-articular, and control group; each 50 patients) and administrated TXA (1g IV and 50mL intra-articular saline, $1 \mathrm{~g}$ intraarticularly and $50 \mathrm{~mL}$ intra-articular saline, and $0 \mathrm{~g}$ and $50 \mathrm{~mL}$ intraarticular saline, respectively). Intra-articular use of TXA reduced more total blood loss $(\mathrm{P}=0.011)$ and reduced more total 48 hours' 
drainage volume than IV use of TXA $(\mathrm{P}<0.001)$. Two patients received a transfusion in the IV and control groups. No deep venous thrombosis or other severe complications had occurred. The hidden blood loss volume had no significant difference among the control, IV, and intra-articular groups (708.6 $\pm 308.2,651.7 \pm 302.9$, and 625.2 $\pm 252.1 \mathrm{~mL}$, respectively; which was $65.6 \%, 70.8 \%$, and $81.1 \%$ of the total loss) [17].

Huang et al. [18] compared 92 patients who received 3 grams of intravenous TXA during TKR with another 92 patients who had 1.5 grams of intra-articular TXA combined with 1.5 grams of intravenous TXA. Interestingly, while both groups had similar effectiveness in reducing blood transfusion rate, patients in the intra-articularintravenous combined group had significantly lesser drainage volume, lesser postoperative knee pain, lesser knee swelling, shorter length of hospital stay, and higher short-term satisfaction [18].

Wang et al. [19] promptly performed a meta-analysis of six prospective randomized controlled trials and cohort studies comprising 679 patients (739 knees) to evaluate the efficacy of intraarticular versus intravenous TXA in primary TKR. They found no significant difference between the two routes of administration in terms of blood loss, blood transfusion requirements, and thromboembolic complications [19].

From the hospital administrators' point of view, the cost savings associated with the use of TXA in TKR is of paramount importance. A recent study by Moskal et al. [20] found that the total direct hospital cost (the combined cost of TXA and blood transfusion) was $\$ 39.14$ / TKR, \$82.59/TKR, and \$84.90/TKR for intra-articular, intravenous, and without TXA respectively. Similarly, the man-hour cost (the time required to successfully deliver a unit of blood and to address transfusion complications) was zero in the intra-articular group as none of the patients required a blood transfusion, as well as 0.007 man-hours/TKR and 0.13 man-hour/TKR for the intravenous and without TXA groups respectively. They concluded that intra-articular TXA has the potential to achieve larger cost-saving and decrease hospital man-hour/TKR [20].

The number of cases was small if compared with some previous studies. Further studies should focus on the following points. First, there is a need to standardize a protocol for the intravenous regimens of TXA (i.e., dosage, timing, and duration of administration) since great variability exists in all studies. Next, there is a lack of a clear, standardized protocol of intra-articular administration of TXA, and relevant studies are needed. Finally, further RCTs should not only improve the study quality but also enlarge the sample size, and this could help to investigate rare complications.

\section{Conclusion}

Injection of TXA effectively reduced postoperative blood loss and so decreased the need for blood transfusion after cemented TKR. This decreased the hospital stay and permits the patients early mobilization instead of bed stay for 3 days. The patient moved after removal or even before removal of the drain 48 hours postoperatively without evidence of the occurrence of complication.

Concerning the administration route, intra-articular administration of TXA seemed to be more effective than intravenous injection in terms of blood loss and transfusion frequency.

\section{References}

1. Kalairajah Y, Simpson D, Cossey AJ, et al. Blood loss after total knee replacement: effects of computer-assisted surgery. The Journal of bone and joint surgery. 2005; 87: 1480-1482.

2. Nadler SB, Hidalgo JU and Bloch T. Prediction of blood volume in norma human adults. Surgery. 1962; 51: 224-232.

3. Cid J and Lozano M. Tranexamic acid reduces allogeneic red cell transfusions in patients undergoing total knee arthroplasty: results of a meta-analysis of randomized controlled trials. Transfusion. 2005; 45: 1302-1307.

4. Ishida K, Tsumura N, Kitagawa A, et al. Intra-articular injection of tranexamic acid reduces not only blood loss but also knee joint swelling after total knee arthroplasty. International orthopaedics. 2011; 35: 1639-1645.

5. Wong J, Abrishami A, El Beheiry $\mathrm{H}$, et al. Topical application of tranexamic acid reduces postoperative blood loss in total knee arthroplasty: a randomized controlled trial. JBJS. 2010; 92: 2503-2513.

6. Rajesparan K, Biant LC, Ahmad M, et al. The effect of an intravenous bolus of tranexamic acid on blood loss in total hip replacement. The Journal of bone and joint surgery. British volume. 2009; 91: 776-783.

7. Zohar E, Ellis M, Ifrach $\mathrm{N}$, et al. The postoperative blood-sparing efficacy of oral versus intravenous tranexamic acid after total knee replacement. Anesthesia \& Analgesia. 2004; 99: 1679-1683.

8. Eubanks JD. Antifibrinolytics in major orthopaedic surgery. JAAOS-Journal of the American Academy of Orthopaedic Surgeons. 2010; 18: 132-138.

9. Salido JA, Marín LA, Gómez LA, et al. Preoperative hemoglobin levels and the need for transfusion after prosthetic hip and knee surgery: analysis of predictive factors. JBJS. 2002; 84: 216-220.

10. Tai TW, Lin CJ, Jou IM, et al. Tourniquet use in total knee arthroplasty: a meta-analysis. Knee Surgery, Sports Traumatology, Arthroscopy. 2011; 19: 1121-1130

11. Tashiro $\mathrm{Y}$, Miura $\mathrm{H}$, Matsuda $\mathrm{S}$, et al. Minimally invasive versus standard approach in total knee arthroplasty. Clinical Orthopaedics and Related Research (1976-2007). 2007; 463: 144-150.

12. Laskin RS, Beksac B, Phongjunakorn A, et al. Minimally invasive total knee replacement through a mini-midvastus incision: an outcome study. Clinical Orthopaedics and Related Research. 2004; 428: 74-81.

13. Kolisek FR, Bonutti PM, Hozack WJ, et al. Clinical experience using a minimally invasive surgical approach for total knee arthroplasty: early results of a prospective randomized study compared to a standard approach. The Journal of arthroplasty. 2007; 22: 8-13.

14. Zhang Y, Fu X, Liu WX, et al. Safety and efficacy of intra-articular injection of tranexamic acid in total knee arthroplasty. Orthopedics. 2014; 37: e775-e782.

15. Hamlin BR, DiGioia AM, Plakseychuk AY, et al. Topical versus intravenous tranexamic acid in total knee arthroplasty. The Journal of arthroplasty. 2015 30: 384-386.

16. Sarzaeem MM, Razi M, Kazemian G, et al. Comparing the efficacy of three methods of tranexamic acid administration in reducing hemoglobin drop following total knee arthroplasty. The Journal of arthroplasty. 2014; 29: 15211524.

17. Wong J, Abrishami A, El Beheiry $\mathrm{H}$, et al. Topical application of tranexamic acid reduces postoperative blood loss in total knee arthroplasty: a randomized, controlled trial. JBJS. 2010; 92: 2503-2513.

18. Huang Z, Ma J, Shen B, et al. Combination of intravenous and topical application of tranexamic acid in primary total knee arthroplasty: a prospective randomized controlled trial. The Journal of arthroplasty. 2014; 29: 2342-2346.

19. Wang $H$, Shen B and Zeng $Y$. Comparison of topical versus intravenous tranexamic acid in primary total knee arthroplasty: a meta-analysis of randomized controlled and prospective cohort trials. The Knee. 2014; 21: 987-993.

20. Moskal JT, Harris RN and Capps SG. Transfusion cost savings with tranexamic acid in primary total knee arthroplasty from 2009 to 2012. The Journal of arthroplasty. 2015; 30: 365-368. 